\title{
Saccadic Behavior during the Response to Pure Disparity Vergence Stimuli I: General Properties
}

\author{
John L. Semmlow \\ Robert Wood Johnson Medical School and \\ Rutgers University \\ Yung-Fu Chen \\ China Medical University \\ Claude Pedrono \\ Essilor International
}

Tara Alvarez

New Jersey Institute of Technology

\begin{abstract}
If two targets are carefully aligned so that they fall along the cyclopean axis, the required eye movement will be symmetrical with the two eyes turning equally inward or outward. When such "pure vergence stimuli" are used only a "pure vergence movement" is required, yet almost all responses include saccadic eye movements, a rapid tandem movement of the eyes. When saccades occur, they must either produce an error in the desired symmetrical response or correct an error from an asymmetrical vergence response. A series of eye movement responses to pure convergence step stimuli (4.0 deg step stimuli) were measured in 12 subjects and the occurrence, timing and amplitude of saccades was measured. Early saccades (within $400 \mathrm{msec}$ of the stimulus onset) appeared in $80 \%$ to $100 \%$ of the responses. In most subjects, the first saccade increased the asymmetry of the response, taking the eyes away from the midline position. In three subjects, these asymmetry-inducing saccades brought one eye, the preferred or dominant eye, close to the target, but in the other subjects these asymmetry-inducing saccades were probably due to the distraction caused by the transient diplopic image generated by a pure vergence stimulus. While many of these asymmetry-inducing saccades showed saccade-like enhancements of vergence, they were, with the exception of two subjects, primarily divergent and did not facilitate the ongoing convergence movement. All subjects had some responses where the first saccade improved response symmetry, correcting an asymmetry brought about by unequal vergence movements in the two eyes. In five subjects, large symmetry-inducing saccades corrected an asymmetrical vergence response, bringing the eyes back to the midline (to within a few tenths of a degree).
\end{abstract}

Keywords: vergence, saccades, oculomotor control, pure vergence, vergence symmetry 


\section{Introduction}

Most eye movements require a combination of version (tandem or conjunctive movements) and vergence (opposition or disjunctive movements); therefore, these components are frequently intermixed in oculomotor movements. When both vergence and version stimuli are present, it is widely held that version asymmetries in the saccadic response assist in moving the eyes disjunctively (Enright, 1986; Zee et al. 1992; Maxwell and King, 1992; Collewijn, Erkelens and Steinman, 1988). Although the two oculomotor components may act collaboratively, they still appear to be independent at the basic control level (Collewijn, Erkelens and Steinman, 1997).

While pure vergence stimuli (i.e., falling exactly along the midline) are rare in everyday life, they are easy to construct in the laboratory and have been used to study the vergence eye movement system. Such stimuli can be presented using a stereo pair of images moving in equal and opposite directions or by two targets placed at different depths along the mid-sagittal (i.e., central or cyclopean) axis. In the latter case, an accommodative (i.e., blur-driven) stimulus may also drive the vergence response. These stimuli are often referred to as "pure vergence stimuli" and might be expected to produce a "pure vergence response," one in which the two eyes rotate an equal amount in opposite directions. Pure vergence responses (which we define here as "symmetrical" vergence responses) would follow along the mid-sagittal plane and there would be no version component to the response.

The presence of a conjunctive movement, particularly saccades, in the response to a pure vergence stimulus would be expected to significantly delay the acquisition of binocular fixation. Yet, saccades are known to occur very frequently in vergence responses (van Leeuwen et al. 1998; Zee et al. 1992; Collewijn, Erkelens and Steinman, 1995). The purpose of this study is to document the prevalence of saccades during pure vergence responses, describe their general behavior, and to present evidence for why these seemly disruptive movements occur.

There are four immediately apparent explanations for the presence of these seemly unnecessary saccades. First, they could be used to bring one eye, likely the "preferred," or "dominant" eye," more quickly to the target (Van Leeuwen, et al., 1998). Embedded saccades might delay the acquisition of full binocular vision, but having one eye on target, particularly the preferred eye, may be sufficient for visual recognition. A second related explanation is that the transient diplopia induced by a pure vergence stimulus produces a compelling saccadic stimulus, particularly if there is a strong ocular preference for one eye. Third, there is considerable evidence that saccades can enhance vergence movements (Maxwell and King, 1992, Collewijn et al. 1988; 1995) through saccade-like burst cell activity that appears to be integrated into the vergence feedback (Busettini and Mays, $2005 \mathrm{~A}$ and B). Such enhancements to vergence may bring both eyes more quickly to the target despite the symmetry error caused by the saccade. Fourth, the saccades could be in response to symmetry errors produced by asymmetrical vergence. Previous work has shown major differences between the speeds at which the two eyes move in a disparity vergence response even in responses free of saccades (Horng et al, 1998). We will present evidence that shows all of these mechanisms are active in all subjects, at least in a few responses, but some subjects exhibit a predominance of one or more of these mechanisms.

\section{Methods}

\section{Definitions}

In any discussion involving both version and vergence eye movements, there are serious semantic problems. Conjunctive movements, also known as version movements, consist of a group of quite different behaviors that appear to be separate at the neurological level. The class of conjunctive eye moments includes: saccades, smooth pursuit, vestibular, and possibly other members. Disjunctive movements, commonly referred to as vergence movements, likewise consist of several movement varieties including fast disparity vergence, slow disparity vergence, and accommodative vergence. These various vergence components are just as separate in behavior and control as saccades, smooth pursuit, etc., but they all carry the term "vergence." Hence vergence stands for both a class of eye movements (i.e., the disjunctive movements) and the various members of that class. Some researchers have used terms "vergence saccades" and "vergence smooth pursuit" to distinguish between two class members, but this can be misleading as conjunctive and disjunctive behaviors and mechanisms are very different.

To remain as close as possible to current terminology, we use the terms version and vergence to describe the class of conjunctive and disjunctive movements. We use 
the somewhat awkward terms "fast disparity vergence" and "slow disparity vergence" to describe two of the members of the class of disjunctive eye movements (along with the unambiguous term accommodative vergence). Under this terminology, "slow vergence" and "slow disparity vergence" refer to different concepts: slow vergence is a behavior that could be due to any number of mechanisms, asymmetric smooth pursuit for example, while slow disparity vergence describes a specific mechanism and underlying neural control process. In summary, vergence without the qualifiers "disparity" or "accommodative" describes only a behavior and unlike the words saccade and smooth pursuit carries no underlying connotation of mechanism.

The standard definitions for version and vergence are:

Vergence $=$ right eye position - left eye position

Version $=($ right eye position left eye amplitude $) / 2$

These definitions apply only to the behavior (and the plots shown here), not to the actual eye movement components. The mix between version and vergence components is much more complex and cannot be isolated by simple addition or subtraction.

\section{Data Acquisition and Subjects}

Twelve subjects between the ages of 21 and 67 participated in this study. All signed informed consent approved by the New Jersey Institute of Technology institutional review board. Eye movements were recorded using an infrared limbus tracking system $(\lambda=950 \mathrm{~nm})$ manufactured by Skalar Iris (model 6500). The manufacturer reports the system has a bandwidth of $200 \mathrm{~Hz}$. All eye movements were well within the system's reported $\pm 25^{\circ}$ linear range. The left and right eye movements were recorded and saved separately. Stimuli were presented using LED targets corresponding to a four degree step change in symmetrical disparity vergence. Each experimental period included approximately 50 convergence and divergence step stimuli presented randomly. The entire experiment typically lasted 30 to 45 minutes. The stimulus began after a 0.5 to $2.0 \mathrm{sec}$. random delay after the subject indicated readiness by a button press. Considerable care was used to align the targets symmetrically along the subject's midline.

The stimuli were LED bars placed at 93 and $46 \mathrm{~cm}$ so during convergence the initial vergence angle was 4 degrees and the final position was 8 degrees. (No effort was made to correct for interpupillary distance.) Divergent responses were also presented, but were not analyzed here. Stimulus presentation occurred after a random delay of 0.5 to 2.0 seconds to reduce anticipatory or predictive mechanism (Alvarez et al. 2002) but was under subject control where the subject indicated readiness by depressing a button. The presentation of stimuli, data acquisition and data storage were controlled by a LabVIEW program. Data acquisition was done at a sampling rate of $200 \mathrm{~Hz}$ which is adequate as we are not interested in the fine structure of saccadic eye movements. Calibration of left and right eye movement responses was performed by recording the output of the eye movement monitor at two known positions before and after each response. Calibration data for each eye were stored with the response and used to construct the eye movement response during offline data analysis.

Analysis

An interactive graphics program written in MATLAB was used to measure saccadic behavior and estimate saccade-induced vergence enhancements. MATLAB software was also used to generate all plots and statistics. Only saccades greater than 0.15 degrees were analyzed in this study. The first step in the analysis was to identify the beginning and end of each saccade in a response. To identify saccades, the version derivative was calculated using the two-point central difference algorithm (Bahill et al., 1982) and the velocity displayed as in Figure 1B. A semi-automated procedure identified the peaks of the saccadic velocity trace and then searched the rising and falling slopes for a sharp change in the derivative. The two ends of the high-velocity segments in the response were then indicated by the '*' points in Figure 1B. These automated identifications were also checked by an operator to ensure correct identification of saccadic onset and offset. In this analysis, we were isolating saccades based on their dynamics: saccades are eye movements that have unique velocity profiles and attain peak velocities much greater than that of other types of eye movement (Bahill $\&$ Stark 1979). From these identifying marks, the position and timing of saccades in the response was determined as indicated with an ' $*$ 'in the version plot of Figure $1 \mathrm{C}$. The saccadic timing identifiers were also used to identify saccadic enhancement to vergence. 


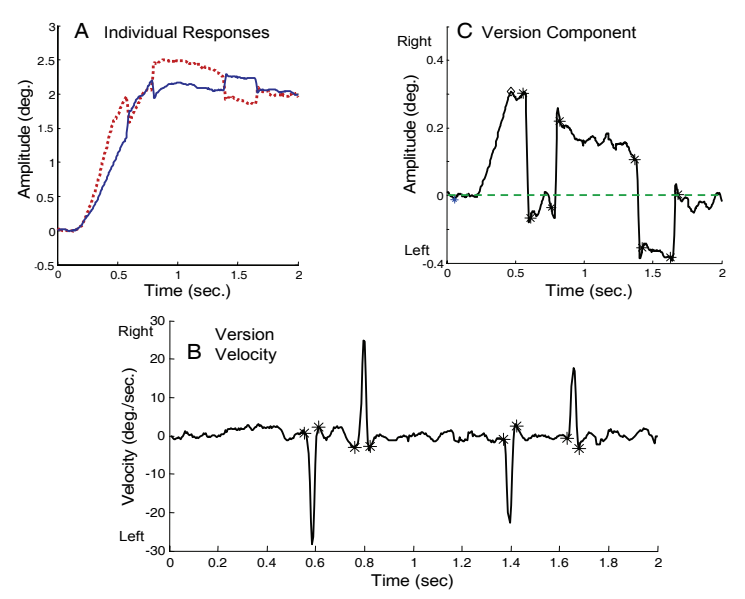

Figure 1 The technique for identifying saccadic time, position, and amplitude information. A) Individual left (dashed red) and right (solid blue) eye movements. B) The velocity trace of the version component showing markers placed to identify the beginning and end of each saccade. C) The time plot of the version component showing the position of the markers obtained from the velocity plot. Saccadic timing information was taken from this plot. The conventions used in this and subsequent figures is to plot convergence as positive for both individual and combined vergence movements as seen in graph $A$ and leftward version movements as negative with the mid-sagittal plane plotted as zero as seen in $C$.

We began by isolating fast and slow components from the two eye movements. First, the derivatives (i.e., velocity curves) of the individual left- and right-eye movements were determined as above. The saccadic markers obtained as in Figure 1 were transferred to these individual eye movement velocities. The high-velocity component of each eye movement was estimated by replacing the saccadic velocity profile with a straight line. This is shown for the left-eye movement in Figure $2 \mathrm{~B}$ where the low-velocity estimate is shown as a dashed red curve and the high-velocity estimate as striped areas. These modified velocity profiles are then integrated to produce highand low-velocity components in each eye. The lowvelocity components are shown for both eyes in Figure $2 \mathrm{C}$, but were not used in this study.

If the high-velocity components could be attributed solely to saccades and the low-velocity to vergence, then this approach would be sufficient to separate out the two components. However, there is considerable evidence that disparity vergence components contain high-velocity components when accompanied by saccades. (Maxwell and King, 1992, Collewijn et al. 1988; 1995). Recently,
Busettini and Mays (2005 A and B) have shown that the high-velocity vergence enhancement that occurs during saccades follows a saccade-like main sequence. They propose that these signals are generated by saccadic burst cells and thus could be thought of as asymmetrical saccades, except that they appear to be integrated into the vergence feedback system. Here we classify all highvelocity movements as a combination of saccades plus any saccade-induced vergence enhancement. The saccade-induced vergence enhancement can be measured as the difference between the left and right saccadic amplitudes after the low-velocity component has been removed. Hence, a saccade-induced vergence enhancement (or saccade asymmetry) is classified in terms of behavior: it is a high-velocity (saccade-like) disjunctive component. This approach avoids the need to affix a specific mechanism (and the resulting controversy) to this vergence component.
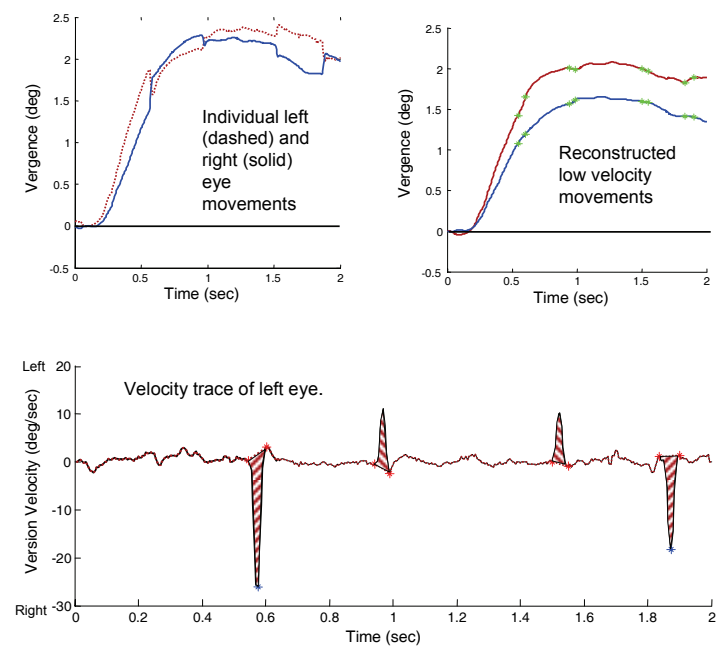

Figure 2 The technique used to separate fast disparity vergence and saccadic components in responses to pure vergence step stimuli. A) Individual eye movements as in Figure $1 A$ and $C$ showing the presence of four saccades. B) The velocity trace of the left eye response (red and black line). The high-velocity contributions have been separated from low-velocity components by linear interpolation between the beginning and end of each saccade (red line ending in '*'). The red shaded area shows the estimated high-velocity components. C) An estimate of the slow velocity eye movements was produced by integrating the vergence velocity trace in $B$ (red line) and a similar operation for the right eye. The high-velocity traces were also integrated to estimate saccadic amplitude and saccade-induced vergence enhancement. Under this strategy, saccadic enhancements to fast disparity vergence are recorded as saccadic asymmetries. 
Journal of Eye Movement Research

$1,(2): 1,1-11$

\section{Measurement of Ocular Motor Dominance}

Subjects viewed an object, a pen, located $40 \mathrm{~cm}$ along their cyclopean midline axis. Using their forefingers and thumbs of each hand, subjects formed a circular aperture in front of each eye to view the object. The subject slowly reduced the diameter of the aperture until they monocularly viewed the object. The eye that the subject used to view the object was noted as their motor dominant eye. This was repeated twice and all subjects reported the same dominance during both trials.

\section{Results}

\section{Examples of Saccades in Responses to Pure Vergence Stimuli}

Figure 3 shows two typical responses to a $4.0 \mathrm{deg}$. convergent step-change in vergence angle. The plots on the left show the individual left and right eye movements (both plotted upwards to aid comparison), the plots on
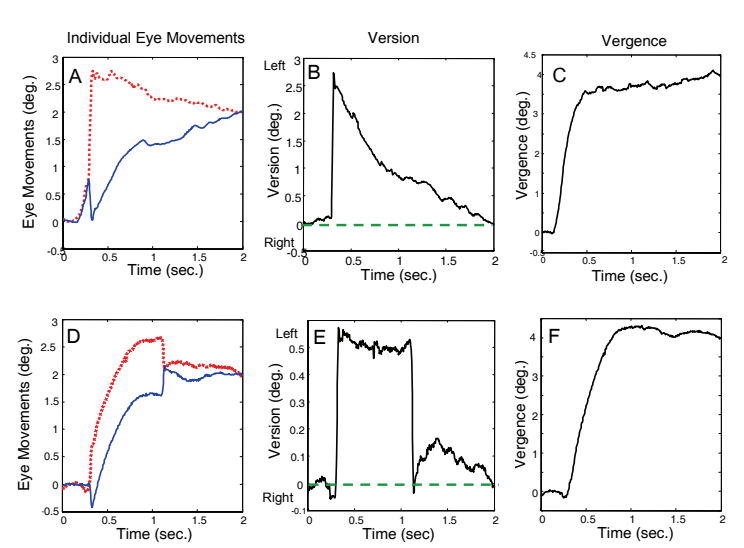

Figure 3 Responses from two subjects to a symmetrical convergent 4.0 deg. step change in stimulus position. In these responses, an early saccade creates a symmetry error. A) and D) Individual left (red dashed line) and right (blue solid line) eye movements showing the presence of saccades along with the vergence response. Convergence is plotted upward for both eyes. B) and E) The version component as defined in Eq. 2. A leftward movement is plotted negative and rightward movement is positive with zero at the mid-sagittal plane. In B, the saccade induced symmetry error is corrected by a slow version movement while in E a second saccade corrects most of the error. $C$ ) and F) The vergence behavior obtained as defined in Eq. 1 with convergence plotted upward. Because the saccades have approximately the same amplitude in both eyes they are difficult to observe when combined into a vergence movement.
Semmlow J, Chen Y-F, Pedrono C, and Alvarez T . (2008)

Disparity Vergence in Response to Pure Disparity Vergence Stimuli the right show the combined vergence response (convergence is plotted as positive), and the center plots show the version component of the response. Version is plotted as negative for leftward movements, positive for rightward movements, with zero at the mid-sagittal plane. Figures 3B and E plot version behavior (as defined in Eq. 2) for two typical responses to a pure vergence stimulus. In an ideal or "pure vergence" response, the response would follow along the mid-sagittal plane and the version component of the response would be zero. The version time course in such a response would be a flat line along the horizontal axis (dashed blue line).

Saccades such as those shown in Figure 3B and E are a common occurrence in responses to pure vergence stimuli. Here we define saccades that increase the symmetry error as "asymmetry-inducing saccades," although they may have an overall visual benefit. "Symmetryinducing" saccades decrease the symmetry error. In Figure 3 , both initial saccades are asymmetry-inducing. In the upper plots the error is corrected by a slow version movement while in the lower plot the error is corrected largely by a second symmetry-inducing saccade. This study will concentrate on the initial saccades while corrective mechanisms are the subject of a later companion paper.
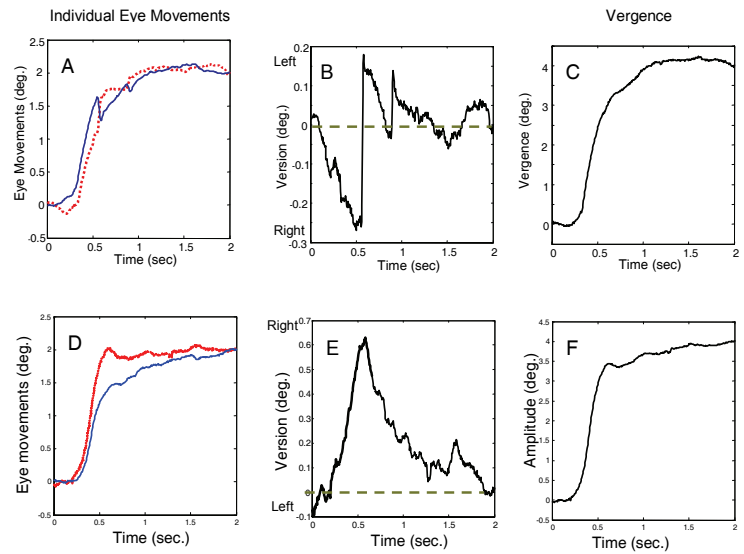

Figure 4 Responses follow the same nomenclature as Figure 3. In these responses, a fast disparity vergence asymmetry creates the symmetry error. A) and D) Individual right (red) and left (blue) eye movements. B) and E) The version component. In B, two saccades appear to be correcting the symmetry error produced by an initial fast disparity vergence asymmetry while in $E$ the fast disparity vergence asymmetry is corrected by a compensatory vergence asymmetry. $C$ and $F$ ) The vergence responses are again smooth due to either saccade cancellation $(C)$ or the absence of saccades $(F)$. 
An example of symmetry-inducing saccades is shown in Figure 4B. In this response, there are two closelyspaced, saccades towards the right visual field that compensate for a fast disparity vergence movement in which the right eye is faster than the left. To round out the picture, Figure 4E shows a saccade-free response in which an initial fast disparity vergence asymmetry is compensated by a later offsetting vergence asymmetry. Such saccade-free responses were uncommon in our untrained subjects.

\section{General Features of Saccades in Vergence Eye Movements}

Nearly all responses contain saccades as shown for our 12 subjects in Figure 5A which shows the percentage of responses with saccade. In fact, 7 of the 12 subjects had saccades in every response. The number of responses analyzed for these subjects is also given in Figure 5A below the subject number. The average number of saccades per response is given in Figure $5 \mathrm{~B}$ and varies among subjects from slightly over 1 saccade per response to almost 4 saccades per response. We attribute the high number of saccades to the fact that our subjects were essentially untrained. We have been able to generate much larger percentages of saccade-free responses, but only in well-trained subjects.
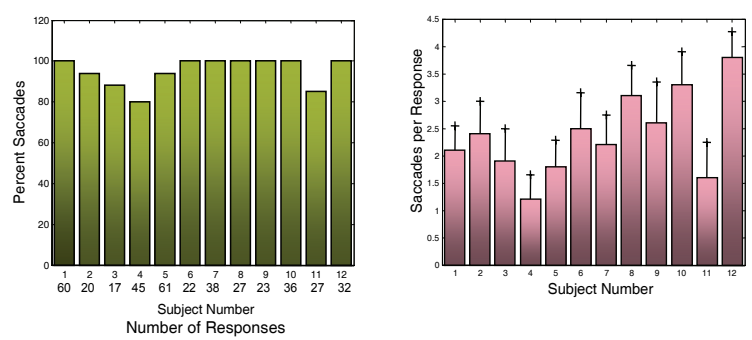

Figure 5 A) Percentage of responses that contain saccades for all 12 subjects. In more than half of our subjects, at least one saccade was found in all responses to pure vergence step stimulus. The number of responses analyzed for each subject is shown below the subject number. B) Saccades per response for our 12 subjects. The number of saccades varies from just over 1 to around 4 saccades per response. The error bars indicate 1 standard deviation.

\section{Asymmetry-inducing Initial Saccades}

While initial saccades can be either symmetryinducing or asymmetry-inducing, for all but two subjects (Subs. 7, and 11) the majority of saccades produce a symmetry error, shown as green (darker) bars in Figure
6A. Initial symmetry-inducing saccades are also present to some extent in all subjects, shown as yellow (lighter) bars Figure 6A. Since these are the first saccades in the response and they are corrective, these responses must contain a vergence-induced symmetry error. Thus, both saccades and asymmetrical fast disparity vergence are active in the generation of symmetry errors in all subjects. The relative importance of each mechanism can be estimated for any given subject by the percentages given in Figure 6A.

Most subjects demonstrate some directional preference in initial saccades particularly in symmetryinducing saccades, Figure 6B. Two subjects (Sub 5 and 9) show initial asymmetry-inducing saccades (green bars) that are relatively evenly distributed in the two directions. However the rest of the subjects showed a directional preference. The saccadic direction that would bring the preferred eye to the target is indicated by the red lines in Figure 6B. The direction of symmetry-inducing saccades (yellow bars) is dependent on the symmetry errors produced by asymmetrical vergence and does not show any consistent pattern in Figure 6B.
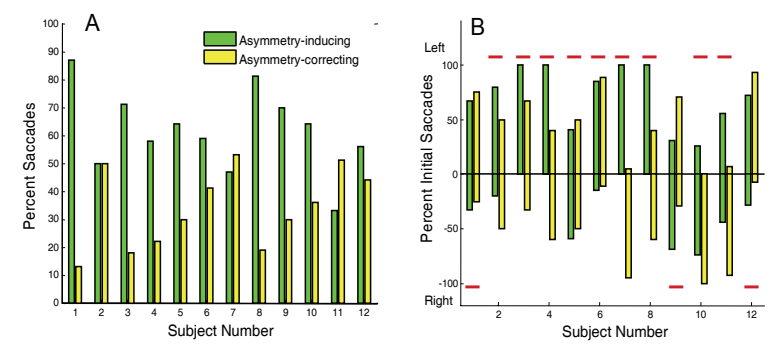

Figure 6 A) The percentage of initial saccades that produce symmetry errors (darker green bars) and the percentage of those that correct symmetry errors (lighter yellow bars). B) Initial direction in percent of error producing (green bars) and error correcting (yellow bars) initial saccades. Percentages are with respect to number of saccades of each type in each direction, not the overall number of initial saccades. The red bars indicate the direction that is toward the preferred (or dominant) eye. (All but three subjects had a preference for the right eye so that a leftward movement would bring that eye closest to the target.)

Preferences in asymmetry-inducing saccades are also evident in the plot of saccadic amplitudes shown in Figure 7. Figure 7 plots the average left and right amplitudes of both initial asymmetry- and symmetry-inducing saccades. A dominance of asymmetry-inducing over symmetry-inducing saccades is seen in most subjects. Several subjects (Subs. 4, 8 and 10) showed a strong directional 
preference in their asymmetry-inducing saccades. Most of our subjects had right eye preference and indeed most of the movement preferences were directed to the left, which would put the right eye closer to the target.

The extent to which asymmetry-inducing saccades bring one eye close to the target is shown in Figure 8 . This plot shows the average position of the eye closest to the target after the end of an asymmetry-inducing saccade; that is, the average position of the left eye (red) after a rightward saccade or the average position of the right eye (blue) after a leftward saccade. The blue and red lines indicate the final target position for the left and right eyes respectively. The size of the diamond reflects the percentage of responses out of all initial saccades that make up the average. For all but one subject (8), both leftward and rightward saccades are too small to bring an eye to the target. However, in three subjects $(4,7,11)$, the preferred right eye is brought to within $0.5 \mathrm{deg}$. of the target, at least in a few responses.

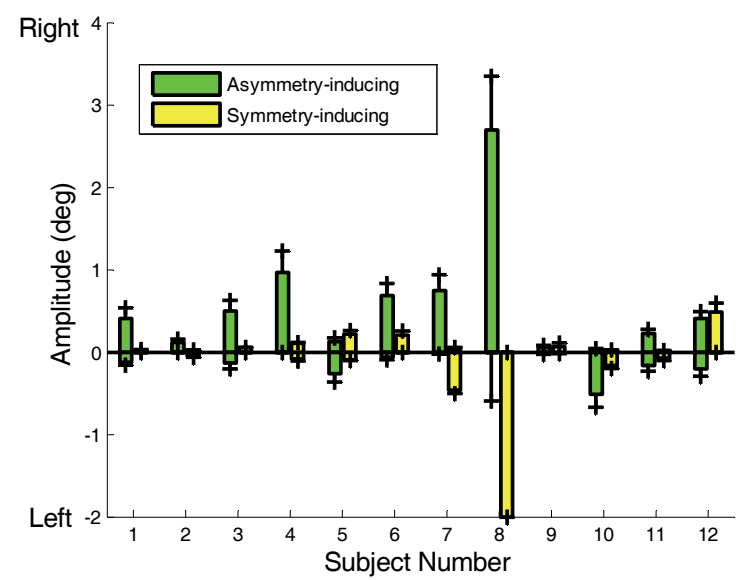

Figure 7 The average amplitude or asymmetry-inducing (green) and symmetry-inducing (yellow) saccades. The marks at the top or bottom of each bar indicate one standard deviation. Over all subjects, the amplitudes of asymmetry-inducing saccades are significantly larger than symmetry-inducing saccades $(p<$ 0.025)

\section{Symmetry-inducing Initial Saccades}

The reduction in symmetry error by symmetryinducing saccades is shown in Figure 9 which plots the average initial and final position of symmetry-inducing saccades. For a number of subjects, the average symmetry error is substantially reduced by these initial saccades so that at the end of the saccade symmetry errors were less than 0.4 deg. In all but one subject (11), the symme-
Semmlow J, Chen Y-F, Pedrono C, and Alvarez T . (2008)

Disparity Vergence in Response to Pure Disparity Vergence Stimuli

try-inducing saccades brought the eyes quite close to the midline and in five subjects $(4,6,7,10$, and 12) these saccades were fairly large, correcting large asymmetries. These large symmetry errors indicate an imbalance between the dynamics of the two eyes during fast disparity vergence (i.e., one eye was faster than the other pulling the alignment point off the mid-sagittal plane). The other subjects had more balanced fast disparity vergence responses so that symmetry-inducing saccades, when they occurred, were small.

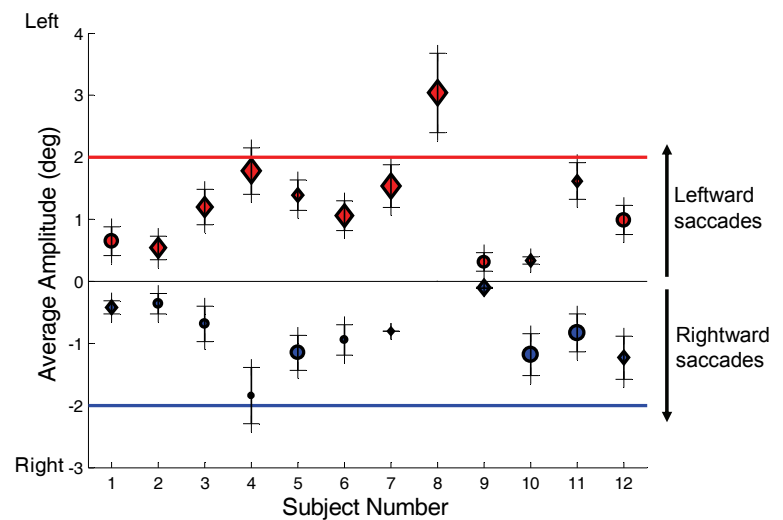

Figure 8 The average position eye closest to the target following an initial asymmetry-inducing saccade; that is, the average position of the right eye (blue) after a leftward saccade and the average position of the left eye (red) after a rightward saccade. The red and blue lines indicate the final target position for the left and right eyes, respectively. The size of the diamond provides an approximate indicator of the percentage of saccades (out of the total number of initial saccades) that contributed to the average. Three subjects $(4,7$, and 11) have initial saccades that place the preferred eye within half a degree of the target.

As might be expected, the symmetry error is significantly larger ( $p<0.005$, paired T-test) when symmetryinducing saccades occur than when asymmetry-inducing saccades occur (Figure 10A). In 7 of 12 subjects, the symmetry error seen when symmetry-inducing saccades occurred was more than double that of when asymmetryinducing saccades occurred. As also shown in Figure 9, in 8 of the subjects, the average symmetry error was larger than 0.5 deg. when an initial symmetry-inducing saccade occurred. Initial asymmetry-inducing saccades also have different timing characteristics than initial symmetry-inducing saccades. Initial asymmetry-inducing saccades occur significantly earlier $(p<0.001)$, approximately 0.2 to $0.3 \mathrm{sec}$ after the stimulus, while initial 
symmetry-inducing saccades occur 0.4 to $0.8 \mathrm{sec}$ after the stimulus (Figure 10B).

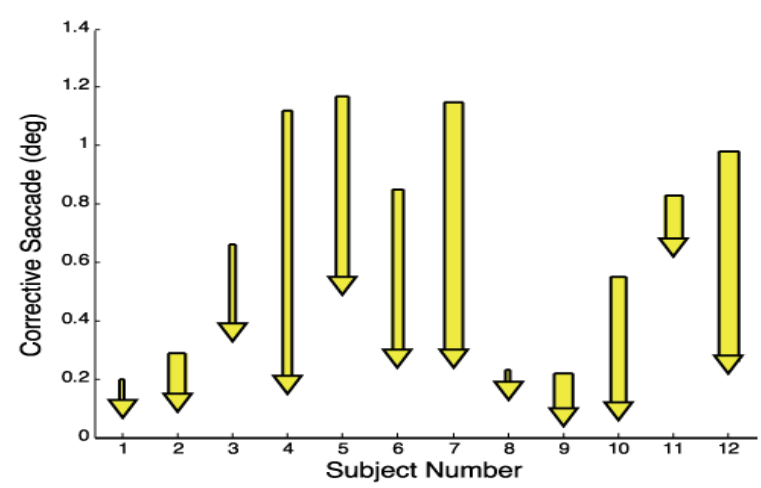

Figure 9 The average correction in symmetry error produced by symmetry-inducing saccades. Bar width indicates the percent symmetry-inducing saccades: $10 \%$ to $50 \%$.
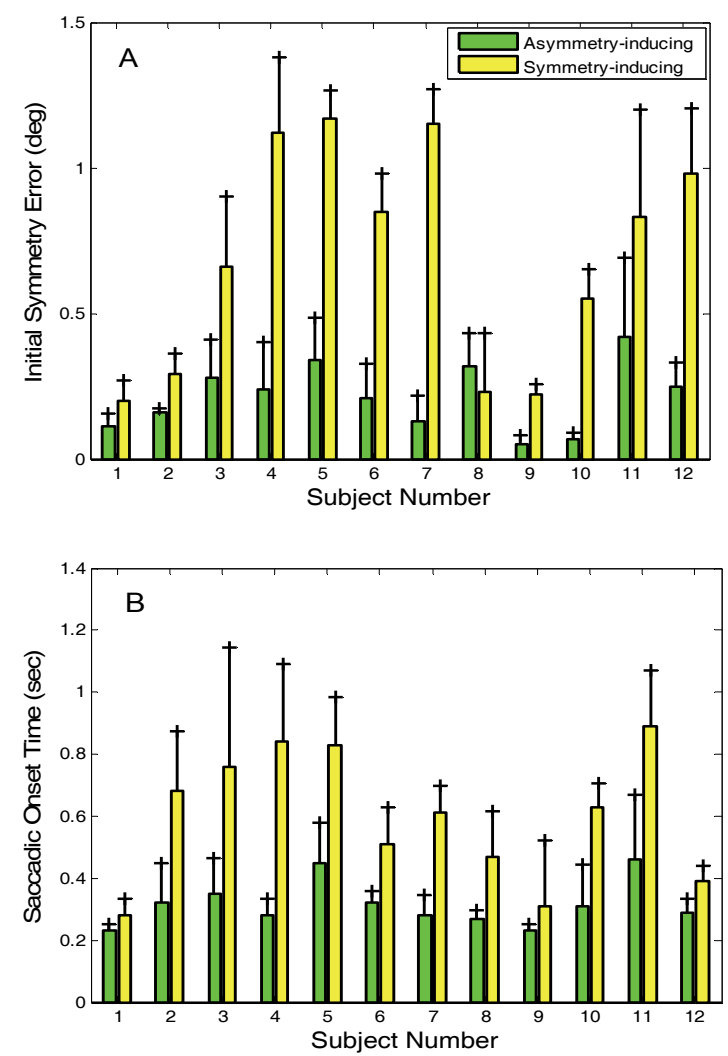

Figure 10 A) Average symmetry errors at onset of the initial saccade for asymmetry-inducing (green bars) and symmetryinducing (yellow bars) saccades. B) Onset time in seconds of asymmetry-inducing (green bars) and symmetry-inducing initial saccades (yellow bars).
Saccade-induced Vergence Enhancements: Saccadic Asymmetry

Saccade-induced enhancements to vergence manifest themselves as asymmetries in the high-velocity component. Saccadic enhancement by initial saccades is summarized for all subjects in Figure 11 which plots the average amplitude of enhancement (obtained by integrating the difference between the high-velocity components of the right- and left-eye movements). Enhancements that improve convergence symmetry are shown as positive while those that increase divergence are shown as negative. For example, if the right eye's high-velocity component was larger than the left eye's during a left-going saccade, the resultant asymmetry would lead to increased convergence and be taken as positive. Similarly, a larger left eye response during a right-going saccade would contribute to divergence and be plotted as negative.

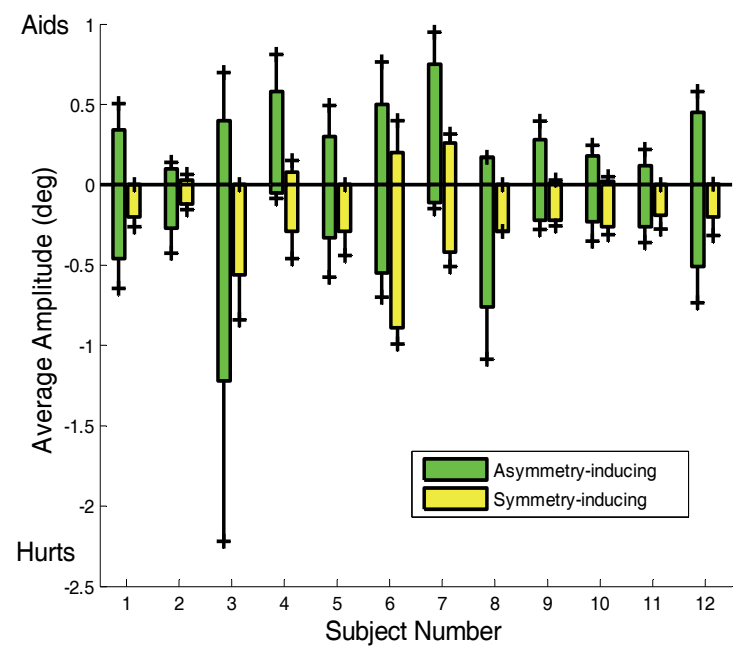

Figure 11 Plot of average enhancement of the vergence response by an asymmetry-inducing and symmetry-inducing saccade. Positive values indicate enhancements that increase convergence, negative values decrease convergence.

Figure 11 shows that saccadic asymmetries are more likely to impede the ongoing convergence (i.e., produce an effective divergence enhancement) in most subjects. Exceptions are found in two subjects (4 and 7) where the average enhancements during asymmetry-inducing (green) saccades add approximately $0.5 \mathrm{deg}$. of convergence. For symmetry-inducing saccades (yellow), the enhancement (i.e., asymmetry) is, on average, counterproductive in that it adds divergence hindering the ongoing convergence response. However these saccades pro- 
Journal of Eye Movement Research

$1,(2): 1,1-11$

vide a useful correction of a vergence-induced asymmetry.

\section{Subject Groups}

In the introduction, four possible mechanisms were provided for the generation of saccades in response to pure vergence stimuli. The first possible explanation was to place one eye close to the desired target and the second was simply the distraction caused by the transient diplopic images. The third possible motivation for these saccades was an enhanced vergence response brought about by the presence of a saccade. The last explanation was to compensate for a vergence asymmetry due to unequal movements in the two eyes. The data of Figures 6 through 11 address these various mechanisms and suggests that for any given subject more than one mechanism may be active. To identify subjects who appeared to exhibit one or more of these mechanisms, the subjects were divided into possible groups using cluster analysis and visual inspection of Figures 6 through 11. The validity of these groups was assessed using an analysis of variance (one-way ANOVA). Cluster analysis was done using the MATLAB "clusterdata" routine that minimized the Euclidian distance between groups. The ANOVA was also implemented in the MATLAB Statistical Toolbox. Since subjects may exhibit more than one saccadic generation mechanism, the groups were determined for each data set and then the subjects were classified based on one or more of these groupings. For all data sets, it was found that dividing the data into more than two groups produced non-significant, single member groups due to the limited number of subjects.

Table I shows the groupings relating to possible saccadic generation mechanisms and their significance as determined by ANOVA. Based on the data showing the closest eye position following a saccade (Figure 8), three subjects were identified as using this strategy. The means of the distance of the closest eye from the target of these three subjects were significantly different from the rest of the group ( $p<0.001)$. This does not mean that other subjects did not use saccades to this end, but their presence is not consistent or common enough to produce statically significant behavior.
Semmlow J, Chen Y-F, Pedrono C, and Alvarez T . (2008) Disparity Vergence in Response to Pure Disparity Vergence Stimuli

Table I Subject Groupings and Associated Mechanisms

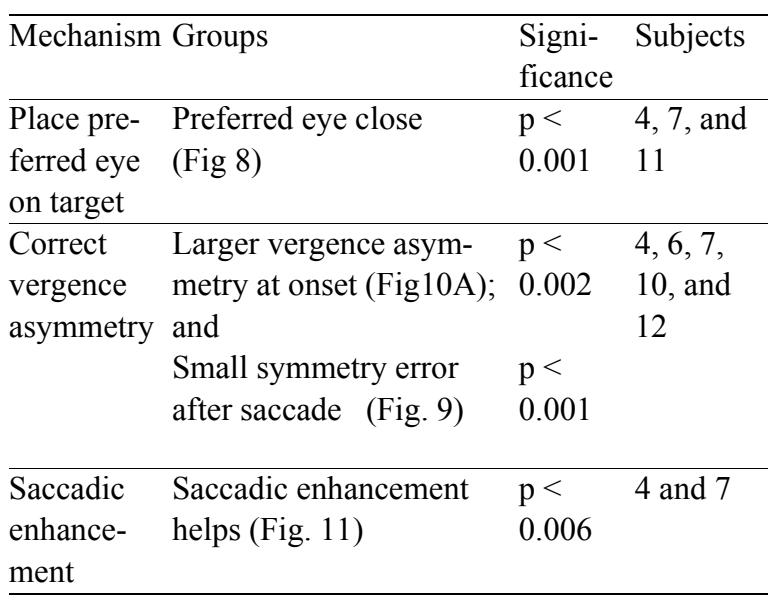

Subjects classified as having saccades that correct asymmetry were identified based on membership in two groups: a group with larger vergence asymmetries at saccadic onset (Figure 10A) and a group with larger corrective saccades (Figure 9). Five subjects had this pattern of groupings. The significance of membership in these two groups (i.e., the difference in means of the relevant data sets were different for these subjects) is also given in the Table. A third class of subjects was identified that may use saccadic enhancement to improve vergence. This class was determined from the enhancement data of Figure 11. Only two subjects were identified as using saccade-like vergence enhancement to aid vergence.

\section{Discussion}

Saccades are found in almost every response to a pure vergence stimulus in our untrained subjects. Our subjects showed averages of between 1.2 to 3.8 saccades per response with most subjects showing averages of approximately two saccades per response. The primary saccades in the response were studied in detail and were found to divide into two categories: saccades that increase symmetry errors and those that reduce this error. Initial asymmetry-inducing saccades occurred earlier (Figure 10B) when disparity vergence symmetries were smaller, usually less than $0.3 \mathrm{deg}$. (Figure 10A). Saccades that reduce symmetry errors occurred approximately 0.2 to $0.4 \mathrm{sec}$ later when the vergence response was well in progress and were more likely to generate an asymmetry. 
An ANOVA-based analysis was used to identify subjects that exhibited behaviors suggesting one of the four saccadic generation mechanisms outlined in the introduction. These subjects had behavioral features that were significantly different from the other subjects. Three subjects appear to be using asymmetry-inducing saccades to bring the preferred eye on to the target at the cost of delayed binocular vision. Two subjects may have benefited from saccadic enhancement of vergence, and five subjects used large symmetry-inducing saccades to offset vergence asymmetries. It is not possible to determine if these mechanisms are truly causal, particularly for saccadic enhancement of vergence. However, there is a strong correlation between the amplitude of symmetryinducing saccades and the initial vergence asymmetry $\left(r^{2}\right.$ $=0.78$, from the data in Figure 9) suggesting a causal relationship. The accurate placement of the preferred eye on the final target found in two subjects also supports causality.

In other stimulus conditions, the saccade-like enhancement of vergence is thought to aid the overall movement. While most subjects showed a saccade-like contribution to vergence, except in two subjects these enhancements were more likely to produce a divergence response that contributes negatively to the ongoing convergence. Transient divergence during saccadic movement has been well documented (Collewijn, et al., 1995) and is likely the result of short-term fusion decay due to the loss of a binocular image during saccadic suppression. This process may be overshadowing any enhancement mechanism in responses to pure vergence stimuli where there is no version component to the stimulus.

No class was established for saccades generated by the distraction of a transient diplopic image, but it is likely that most initial asymmetry-inducing saccades fall into this class. Until the vergence system responds, a pure vergence stimulus will produce two disparate images in each eye. If one eye is even slightly dominant, the stimulus to make a saccade toward the more intense image is quite strong. This is seen in four of our subjects $(3,4,7$, and 8) who made all of their initial asymmetry-inducing saccades favoring the preferred eye, figure $6 \mathrm{~B}$.

The existence of four different motivations for saccades may account for the prevalence of saccades in responses to pure vergence stimuli. If the subject avoids an early asymmetry-inducing saccade, subsequent vergence movements may generate symmetry errors which would stimulate a saccade. Since saccade-free vergence responses are often highly asymmetrical (Horng et al., 1998), a saccade due to one process or the other is almost guaranteed.

Since the vergence stimulus was a real target (i.e., not present through a haploscope), accommodative vergence would also be active. Previous studies comparing the dynamics of responses to binocular and disparity-only stimuli (produced by a haploscope) have shown that accommodative vergence contributes a very small role, particularly in the early response (Hung et al., 1987). This is due to the fact that the accommodative system has a much longer latency than disparity vergence and produces slower responses. If accommodative vergence has any overt influence on the vergence motor behavior, it would manifest at the end of the movement long after the occurrence of the initial saccades described here. LED target stimuli were used because we were working with untrained subjects where eye movements to real targets are more synergistic to real world conditions.

Despite symmetry deviations the eyes always arrive at the correct final position: at the end of the movement (1.0 to $2.0 \mathrm{sec}$ after the stimulus), the symmetry error is essentially zero (of the order of minutes of arc). This implies that symmetry errors produced by initial saccades must be compensated either by subsequent saccades and/or by an offsetting asymmetry in the vergence response. Krommenhoek and Van Gisbergen (1994) have shown that both saccades and vergence components use internal (i.e., extra-retinal) feedback loops in their control. These internal feedback mechanisms may provide the requisite compensatory processes. The correction of symmetry error is the subject of a subsequent companion paper.

\section{Conclusion}

The vast majority of responses to a pure vergence stimulus contain saccades. Considering only the first saccades in the response, the majority produce an error in symmetry, a deviation away from the mid-sagittal plane. The evidence presented here suggests that four different mechanisms provide a stimulus to these saccades. About $15 \%$ to $50 \%$ (depending on the subject) of the initial saccades were correcting a deviation produced by disparity vergence bringing the eyes closer to the midsagittal plane where they must end up by the end of the movement. Many of the initial asymmetry-inducing saccades were in a direction that brought the preferred 
Journal of Eye Movement Research

$1,(2): 1,1-11$

(i.e., dominant eye) closer to the target and in three subjects the preferred eye was within a half degree of the target. The possibility that saccadic enhancement is being used to aid the ongoing vergence was seen in only two of the 12 subjects.

\section{Acknowledgements}

Yung-Fu Chen was support, in part, by a grant CMU96-145 from China Medical University, Taichung, Taiwan. John Semmlow and Tara Alvarez were supported, in part, by a grant from Essilor International, St. Maur, FRANCE.

\section{References}

Alvarez TL, Semmlow JL, Yuan W, Munoz P (2002) Comparison of Disparity Vergence System Responses to Predictable and Non-predictable Stimulations. Curr. Psych. Cog., 21, 243-261.

Bahill, AT, Kallman, JS, Lieberman, JE (1982). Frequency limitations of the two-point central difference differentiation algorithm. Biol.Cybern., 45, 1-4.

Bahill AT, and Stark L (1979) The trajectories of saccadic eye movements. Sci Am 240(1) 108-117.

Busettini C and Mays, L. (2005A) Saccade-vergence interactions in Macaques. I Test of the omnipause multiply model. J. Neurophysiol. 94:2295-2311.

Busettini C and Mays, L. (2005B) Saccade-vergence interactions in Macaques. I Vergence enhancement as the product of a local feedback vergence motor error and a weighted saccadic burst. J. Neurophysiol. 94:23122330 .

Collewijn, H. Erkelens, CJ and Steinman R. (1988) Binocular co-ordination of human horizontal saccadic eye movements. J Physiol. 404:157-182.

Collewijn, H. Erkelens, CJ and Steinman R. (1995) Voluntary binocular gaze-shifts in the plane of regard: dynamics of version and vergence. Vision Res. 35:3335-3358.

Collewijn, H. Erkelens, CJ and Steinman R. (1997) Trajectories of the human binocular fixation point during conjugate and non-conjugate gaze-shifts. Voluntary binocular gaze-shifts in the plane of regard: dynamics of version and vergence. Vision Res. 37:1049-1069.
Semmlow J, Chen Y-F, Pedrono C, and Alvarez T . (2008) Disparity Vergence in Response to Pure Disparity Vergence Stimuli

Enright, JT Facilitation of vergence changes by saccades: Influences of misfocused images and of disparity stimuli in man. (1986) J. Physiol. 371:69-87.

Horng J-L, Semmlow JL, Hung, GK, Ciuffreda, KJ. (1998) Dynamic Asymmetries in Disparity Vergence. Vision Res. 38:1254-1261.

Hung G, Semmlow JL and Ciuffreda K: (1983) Identification of Accommodative Vergence Contribution to the Near Response Using Response Variation. Invest Ophthal 24:772-777.

Krommenhoek, KP and Van Gisbergen, JAM (1994) Evidence for nonretinal feedback in combined version-vergence eye movements. Exp Brain Res. 102:95-109.

Maxwell, JS and King WM. (1992) Dynamics and efficacy of saccade-facilitated vergence eye movements in monkeys. J. Neurophysiol. 68:1248-1259.

Van Leeuwen AF, Collewijn H, Erkelens CJ (1998) Dynamics of horizontal vergence movements: interaction with horizontal and vertical saccades and relation with monocular preferences. Vision Res. 38(24) 3943-54.

Zee DS, Fitzgibbon EJ and Optican LM (1992) Saccadevergence interactions in humans. J. Neurophysiol 68(5) 1624-1641. 